\title{
Wie Kunden entscheiden
}

\section{Grit Koch}

Eine neue Studie untersuchte, aus welchen Gründen sich Nutzer für einen bestimmten ambulanten Pflegedienst entscheiden. Eine mögliche Konsequenz der Ergebnisse könnte die Schaffung einer hochwertigen Dienstleistungsmarke sein, die das Vertrauen der Kunden in deren Anbieter deutlich erhöhen kann.

Nach welchen Kriterien wählt ein Nachfrager ambulanter Pflegedienstleistungen aus einer Vielzahl von möglichen Alternativen seinen zukünftigen Pflegedienst aus? Eine möglichst detaillierte Antwort auf diese Frage dürfte, gerade mit Blick auf das Persönliche Budget, für jeden Anbieter langfristig existenzsichernd sein. Besonders aufschlussreich dürften solche Erkenntnisse sein, die sich aus direkter Quelle, also aus der Befragung von Nutzern, ableiten lassen.

Eine solche Klientenbefragung wurde im Rahmen einer Diplomarbeit zum Thema »Marken und Markenbewusstsein in der Sozialen Arbeit « durchgeführt. Teilgenommen haben zirka 250 Nutzer von acht ambulanten Pflegediensten in und um Neubrandenburg, einer Stadt in Mecklenburg-Vorpommern mit rund 70.000 Einwohnern. Die Verteilung der Fragebögen an die Nutzer erfolgte über die Pflegekräfte des jeweiligen betreuenden Pflegedienstes. Insgesamt beteiligten sich acht von insgesamt zehn Neubrandenburger Pflegediensten in dieser indirekten Form an der Befragung. Bei der Verteilung wurden all diejenigen Klienten berücksichtigt, die Leistungen nach dem SGB V oder SGB XI erhielten. Insgesamt wurden damit rund 500 Fragebögen verteilt.

\section{Entscheidungskriterien}

Innerhalb der Befragung, in der Mehrfachnennungen möglich waren, gaben 38 Prozent der Befragungsteilnehmer an, dass sie sich aufgrund einer Notsituation schnell entscheiden mussten. Fast genauso viele, nämlich 35 Prozent, benannten den guten Ruf ihres Pflegedienstes als Entscheidungskriterium. Die Empfehlung durch den Arzt gaben 32 Prozent der Befragten als relevant für ihre Entscheidung an. Bei 27 Prozent der Befragten wurde die Auswahl des Pflegedienstes mehr oder minder von einem Angehörigen vorgenommen. Genauso häufig spielte die

Grit Koch studierte Sozialpädagogik an der Fachhochschule Neubrandenburg. In ihrer von Prof. Dr. Bernd Halfar betreuten Diplomarbeit zum Thema »Marken und Markenbewusstsein in der Sozialen Arbeit untersuchte sie die Gründe, warum sich die Nutzer von ambulanten Pflegediensten für einen bestimmten Anbieter entscheiden.
Empfehlung durch Bekannte oder Verwandte eine Rolle. Schon vorher gute Erfahrungen mit dem gewählten Pflegedienst haben 22 Prozent der Befragten gemacht. Ebenso viele Befragte haben angegeben, dass ihnen der gewählte Pflegedienst (weltanschaulich) sympathisch sei. Die Antwortalternative »andere Gründe« wurde von 16 Prozent der Befragten angekreuzt. 12 Prozent der Befragungsteilnehmer gaben an, dass für sie bei der Entscheidung eine bestimmte Leistung wichtig war. 10 Prozent der Teilnehmer haben sich auch aufgrund finanzieller Überlegungen für ihren Pflegedienst entschieden, 7 Prozent gaben Werbung als Entscheidungskriterium an.

\section{Entscheidungsformen}

Nach dem Überblick über den Einfluss der verschiedenen Entscheidungskriterien bei den Befragungsteilnehmern soll genauer auf die Art der getroffenen Entscheidung geschaut. In der Befragung gaben 223 Teilnehmer eine gültige Antwort auf die entsprechende Frage. Mehr als die Hälfte der Befragten, nämlich 53 Prozent, gaben an, dass sie von Anfang an zum gewählten Pflegedienst wollten. Für 30 Prozent der Befragten war die Entscheidung mehr oder minder Zufall. Die übrigen gegebenen Antworten verteilten sich fast gleichmäßig auf die Alternativen »Ich habe mich zwischen mehreren Pflegediensten entschieden « und »Ich weiß nicht genau.«

Mit insgesamt 38 Prozent der Befragungsteilnehmer hat ein durchaus beachtenswerter Anteil der Befragten angegeben, dass er sich aufgrund einer Notsituation entschieden hat. Wegen der damit verbundenen Notwendigkeit einer schnellen Entscheidungsfindung liegt die Vermutung nahe, dass innerhalb einer Notsituation weniger von Anfang an bewusste Entscheidungen als ohne ihr Vorhandensein getroffen werden.

Tatsächlich haben sich nur 41 Prozent derjenigen Befragten, die ihre Entscheidung innerhalb einer Notsituation getroffen haben, von Anfang an bewusst für ihren Pflegedienst entschieden, gegenüber 59 Prozent der übrigen Befragten. Noch deutlicher zeigen sich die Unterschiede zwischen den beiden Gruppen in Bezug auf die zufällige Entscheidungsfindung: 45 Prozent derjenigen, die sich aufgrund einer Notsituation entschieden haben und nur 21 Prozent der Vergleichsgruppe gaben an, sich mehr oder minder zufällig für ihren Pflegedienst entschieden zu haben. Etwa gleich hoch ist dagegen der jeweilige Anteil derjenigen, die zwischen mehreren Pflegediensten hin- und herüberlegt haben und derjenigen, die angegeben haben, dass sie die Art der Entscheidung nicht mehr genau einschätzen können. 
Betrachtet man das Abwägen zwischen mehreren Pflegediensten als bewusste Entscheidung und das »Nicht-Genau-Wissen« als Indiz für eine eher zufällige Wahl (da man sich nicht mehr erinnern kann) bedeutet das, dass situation befunden haben, gegenüber 68 Prozent der Vergleichsgruppe, bewusst für ihren Pflegedienst entschieden haben.

Optimal aus der Sicht eines Anbieters dürften solche Nachfrager sein, die sich von Anfang für ihn und für seine Leistungen entscheiden. Ein hoher Anteil dieser Entscheidungsform an den insgesamt getroffenen Entscheidungen trägt viel zur Wettbewerbsfähigkeit und damit zur relativen Planungssicherheit eines Pflegedienstes bei. Damit kommt denjenigen Entscheidungskriterien, die im Hinblick auf eine von Anfang an bewusst getroffene Entscheidung ausschlaggebend sind, aus Marketinggesichtspunkten eine besondere Bedeutung zu. Um in der Befragung überprüfen zu können, ob sich Aussagen speziell für sich die Hälfte derjenigen Befragten, die sich in einer Not-

Die übrigen Entscheidungskriterien weisen keine Besonderheiten im Hinblick auf ihre Bedeutung für die Entscheidungsformen auf.

\section{Einfluss einzelner Entscheidungskriterien}

Da im Zusammenhang mit den für die Entscheidung relevanten Kriterien Mehrfachnennungen möglich waren, ergibt sich die Frage, welchen Einfluss die einzelnen Entscheidungskriterien für das Zustandekommen einer von Anfang an bewusst getroffenen Entscheidung haben. Sind einige Entscheidungskriterien besonders relevant für ihr Zustandekommen, während andere nur im Zusammenhang mit weiteren Kriterien zu einer bewussten Entscheidung führen?

Tatsächlich sprechen die Ergebnisse durchaus für eine unterschiedliche Wirkung der einzelnen Entscheidungskriterien. Die Empfehlungen durch Dritte werden zwar häufig als Entscheidungshilfen herangezogen, bedingen aber dabei oftmals nur im Zusammenhang mit den Kriterien »guter Ruf «, »weltanschauliche Sympathie« und »vorherige gute Erfahrungen« eine bewusste Entscheidung. Diese drei Entscheidungskriterien führen dagegen

diese Art von Entscheidung treffen lassen, wurden zwei Gruppen gegenübergestellt: die erste bestehend aus denjenigen Befragten, die von Anfang an zu ihrem Pflegedienst wollten und die zweite bestehend aus denjenigen Befragungsteilnehmern, die sich mehr oder minder zufällig entschieden haben.

41 Prozent derjenigen, die sich bewusst für ihren Pflegedienst entschieden haben, nannten den guten Ruf ihres Pflegedienstes als Entscheidungskriterium, dagegen führten nur 20 Prozent der Befragungsteilnehmer, die eine zufällige Entscheidung getroffen haben, dieses Kriterium an. Noch deutlichere Unterschiede werden bei den vorher gemachten guten Erfahrungen deutlich: 36 Prozent derjenigen, die sich bewusst entschieden haben, betonten deren Relevanz, gegenüber 9 Prozent derjenigen, die sich zufällig entschieden haben. Dass sie ihren Pflegedienst (weltanschaulich) sympathisch fanden, gaben 25 Prozent derjenigen an, die sich bewusst und nur 14 Prozent derjenigen, die sich zufällig entschieden haben als Entscheidungskriterium an. Ebenfalls anteilig höher als bei den zufälligen Entscheidungen (21 Prozent) ist bei den bewussten Entscheidungen der Einfluss durch Bekannte und Verwandte (27 Prozent).

Neben Entscheidungskriterien, die bei bewussten Entscheidungen anteilig höher als bei zufälligen Entscheidungen auftreten, gibt es ebenfalls Kriterien, bei denen es sich genau umgekehrt verhält. 53 Prozent der zufälligen Entscheidungen standen im Zusammenhang mit einer Notsituation, gegenüber 27 Prozent der bewussten Entscheidungen. Auch eine Empfehlung durch den Arzt tritt eher im Zusammenhang mit einer zufälligen (49 Prozent) als mit einer bewussten Entscheidung auf (25 Prozent). Die Angehörigenentscheidung hatte bei einer zufälligen Entscheidung mit 33 Prozent größere Relevanz als bei einer bewussten Entscheidung mit 19 Prozent. auch schon unabhängig voneinander zu einem hohen Anteil bewusster Entscheidungen, besonders hoch ist dieser im Zusammenhang mit vorher gemachten guten Erfahrungen mit dem Pflegedienst.

\section{Betrachtungen}

Die Ergebnisse der Befragung zeigen: Die meiste Relevanz in einer bewusst getroffenen Entscheidung hat der gute Ruf eines Pflegedienstes; die (weltanschauliche) Sympathie, die ihm entgegengebracht wird, und die vorherigen guten Erfahrungen mit dem Pflegedienst.

Auch wenn die Ergebnisse nur für den untersuchten (regionalen) Bereich Aussagekraft besitzen, sprechen doch mehrere Gründe dafür, dass die herausgestellten Entscheidungskriterien auch darüber hinaus von Bedeutung sind: Die vorher gemachten guten Erfahrungen mit einem Pflegedienst bieten dem Nachfrager — vor dem Hintergrund des Mangels an rationalen Entscheidungsmöglichkeiten — eine gute Möglichkeit, von der Qualität der gemachten Erfahrungen auf die Qualität zukünftiger Leistungen zu schließen. Wenn ein derartiger Rückschluss natürlich auch nicht immer richtig sein muss, ist doch davon auszugehen, dass ein Nachfrager, der vorher gute Erfahrungen mit einem Pflegedienst gemacht hat, sich bei seiner Entscheidung wohler fühlen wird als jemand, der solche Erfahrungen nicht gemacht hat und auch keine gleichwertigen Entscheidungskriterien heranziehen kann.

Wenn es also einem Pflegedienst gelingt, eine Vielzahl von direkten Berührungspunkten zwischen ihm und möglichen späteren Nutzern zu schaffen, wird sich die Wahrscheinlichkeit erhöhen, dass sich die dabei gemachten Erfahrungen, vorausgesetzt natürlich, sie sind positi- 
ver Art, später in einer bewussten Entscheidung für diesen Pflegedienst auswirken. Allerdings können derartige Bemühungen nicht die einzigen bleiben: Zum einen wird es immer Nachfrager geben, die auf diesem Weg nicht erreicht werden können, zum anderen erscheint es wahrscheinlich, dass eine gleichzeitige Konzentration auf die Förderung weiterer Entscheidungskriterien den Anteil bewusster Entscheidungen noch erhöhen kann.

Die Bedeutung des guten Rufes eines Pflegedienstes und der (weltanschaulichen) Sympathie, die ihm entgegengebracht wird, ist wohl am besten dadurch zu erklären, dass es sich - wie auch bei den vorherigen Erfahrungen - um Entscheidungskriterien aktiver Art handelt, also um Kriterien, die durch die Entscheidungsträger selber wahrnehmbar und erlebbar sind. Der gute Ruf einer Einrichtung entsteht, auch wenn er über verschiedene Informationsquellen zustande kommt, aus und in der Wahrnehmung desjenigen, der ihn benennt. Die (weltanschauliche) Sympathie ist ebenfalls ein eigenes Gefühl, eine eigene Wahrnehmung. Diese beiden Kriterien zeigen deutlich, dass dort, wo rationale Entscheidungskriterien nicht oder nur bedingt zur Verfügung stehen, emotionale Faktoren an Bedeutung gewinnen.

Findet die emotionale Komponente im Marketing eines Anbieters keine Berücksichtigung, kann der Mangel an rationalen Entscheidungsmöglichkeiten beim Nachfrager leicht zu Unsicherheiten und Ängsten führen — schließlich basiert seine Entscheidung letztendlich »nur « auf einem Leistungsversprechen des Pflegedienstes, von dem er nicht wissen kann, ob es wirklich so eingehalten wird. Ein Anbieter, der möchte, dass sich ein Großteil der Nachfrager bewusst und somit kalkulierbar für seinen Pflegedienst entscheidet, hat gute Chancen, wenn er dem Nicht-Wissen-Können des Nachfragers im Hinblick auf die Leistung selber und auf die Richtigkeit der Entscheidung eine eben so starke emotionale Komponente entgegensetzt. Aus dem Nicht-Wissen-Können wird dann ein Vertrauen-Können.

Bei der Schaffung eines derartigen Vertrauensverhältnisses kann eine qualitativ hochwertige Dienstleistungsmarke eine äußerst wichtige Rolle spielen. Dieser gelingt es nämlich nicht nur, den Bedürfnissen des Nachfragers im Hinblick auf die Leistung selber, sondern auch im Hinblick auf die Entscheidungsfindung gerecht zu werden. Dadurch ist es dem Nachfrager möglich, sich trotz minimaler Informationskosten - und diese minimalen Informationskosten sind besonders im Falle einer erforderlichen schnellen Entscheidung aufgrund einer Notsituation wichtig - mit einem guten Gefühl bewusst für eine Leistung zu entscheiden. Eine Marke, die solches zu erreichen vermag, muss natürlich mehr Komplexität aufweisen als ein Markenzeichen. Obwohl diesem eine wichtige (Markierungs-) Funktion zukommt, schließt es sich damit aus, Dienstleistungsmarke und Markenzeichen oder Logo gleichzusetzen. Vielmehr soll die Marke als »unverwechselbares Leistungsprofil mit Vertrauensvorschuss« (Bernd Halfar) verstanden werden.

\section{Resümee}

Warum sich die Nachfrager gerade für eine bestimmte Dienstleistungsmarke entscheiden sollten, kann in dreifacher Weise beantwortet werden:

- Die Dienstleistungsmarke basiert auf einem unverwechselbaren Leistungsprofil, das besser als alle vergleichbaren Alternativen dazu geeignet ist, die Bedürfnisse der Nachfrager und späteren Nutzer zu erfüllen.

- Die Leistungskomponenten, die diese Unverwechselbarkeit und damit den Wettbewerbsvorteil ausmachen, werden schon vor der Inanspruchnahme in Form eines Leistungsversprechen für den Nachfrager wahrnehmbar herausgestellt.

- Dem Nachfrager und späteren Nutzer ist es möglich, einen unmittelbaren Zusammenhang zwischen Leistungsversprechen und Leistung herzustellen. Konkret ausgedrückt kann er darauf vertrauen, dass die Leistung so ausgeführt wird, wie durch den Anbieter im Vorhinein versprochen. Seine späteren Erfahrungen mit der Leistung bestätigen und verstärken dieses Vertrauen.

In diesem dritten Punkt liegt die interessanteste aber auch schwierigste Herausforderung an eine Marke. Ein Leistungsversprechen wird nämlich nur dann als glaubwürdig wahrgenommen werden, wenn alle Eindrücke und Erfahrungen, die der Nachfrager unmittelbar (im direkten Kontakt) oder mittelbar (über Dritte) im Zusammenhang mit der Leistung oder deren Anbieter macht, dieses Leistungsversprechen bestätigen. Jeder mögliche Berührungspunkt mit einem Unternehmen kann sein Image und damit seine Glaubwürdigkeit beeinflussen und ist somit markenrelevant.

In der Entstehungsphase einer solchen Dienstleistungsmarke kann eine Befragung von Nachfragern oder (potenziellen) Nutzern wichtige Hinweise im Hinblick auf deren Bedürfnisse im Hinblick auf die Leistungserstellung und die Entscheidungsfindung bringen. 